\title{
In silico modeling of the Moniliophthora perniciosa Atg8 protein
}

\author{
A.C.F. Pereira ${ }^{1}$, T.H.S. Cardoso $^{2}$, M. Brendel $^{1}$ and C. Pungartnik ${ }^{1}$ \\ ${ }^{1}$ Laboratório de Biologia de Fungos, Centro de Biotecnologia e Genética, \\ Universidade Estadual de Santa Cruz, Ilhéus, BA, Brasil \\ ${ }^{2}$ Departamento de Bioquímica, Universidade Federal de São Paulo, \\ São Paulo, SP, Brasil \\ Corresponding author: M. Brendel \\ E-mail: martinbrendel@yahoo.com.br
}

Genet. Mol. Res. 12 (4): 6619-6628 (2013)

Received June 21, 2013

Accepted October 17, 2013

Published December 11, 2013

DOI http://dx.doi.org/10.4238/2013.December.11.13

\begin{abstract}
Autophagy is defined as an intracellular system of lysosomal degradation in eukaryotic cells, and the genes involved in this process are conserved from yeast to humans. Among these genes, $A T G 8$ encodes a ubiquitin-like protein that is conjugated to a phosphatidylethanolamine (PE) membrane by the ubiquitination system. The Atg8p-PE complex is important in initiating the formation of the autophagosome and thus plays a critical role in autophagy. In silico modeling of Atg8p of Moniliophthora perniciosa revealed its three-dimensional structure and enabled comparison with its Saccharomyces cerevisiae homologue ScAtg8p. Some common and distinct features were observed between these two proteins, including the conservation of residues required to allow the interaction of $\alpha$-helix 1 with the ubiquitin core. However, the electrostatic potential surfaces of these helices differ, implying particular roles in selecting specific binding partners. The proposed structure was validated by the programs PROCHECK 3.4, ANOLEA, and QMEAN, which demonstrated 100\% of amino acids located in favorable regions with low total energy. Our results showed that MpAtg8p contains the same functional domains
\end{abstract}


(3 $\alpha$-helices and $4 \beta$-sheets) and is similar in structure as the ScAtg8p yeast. Both proteins have many conserved sequences in common, and therefore, their proposed three-dimensional models show similar configuration.

Key words: Basidiomycetes; Autophagosome; Protein homology

\section{INTRODUCTION}

Eukaryotic cells employ autophagy (ATG, a general term for catabolic processes involving the lysosomal/vacuolar pathway) to degrade damaged or obsolete organelles and proteins. ATG is an evolutionarily conserved mechanism for degradation of biomolecules and is generally defined as a lysosome-dependent mechanism of intracellular degradation that is used for the turnover of cytoplasmic macromolecules in starving cells (Yorimitsu et al., 2007). Three forms of autophagic pathways have been described, chaperone-mediated autophagy (independent of vesicle formation), macro- and micro-autophagy (dependent on vesicles) in plants, mammals and fungi (Shpilka et al., 2011; Li and Vierstra, 2012). Both macro- and micro-autophagy involve the movement of membranes. Whereas in micro-ATG the transport of material destined for degradation occurs via invagination of the lysosomal membrane, macro-ATG involves the formation of double membranes of autophagic vacuoles, also known as autophagosomes, which transport cytoplasmic cargo to lysosomes for degradation (Shpilka et al., 2011; Li and Vierstra, 2012).

In filamentous fungi, ATG is involved in different cellular pathways such as cellular degradation, nutrient recycling, cellular differentiation, and cell death (Pinan-Lucarré et al., 2003; Pollack et al., 2009). Pinan-Lucarré et al. (2003) showed that ATG-mutants of Podospora anserina had compromised differentiation due to the absence of internal nutrient supply, lacking provision of new 'building blocks' for cellular differentiation while ATG-mutants of Aspergillus nidulans had abnormal development of conidiophores, even though forming aerial hyphae (Kikuma et al., 2006).

Molecular studies on ATG began more than a decade ago, starting primarily from genetic screenings in the budding yeast Saccharomyces cerevisiae and the methylotrophic yeasts Pichia pastoris and Hansenula polymorpha (Suzuki and Ohsumi, 2007; He and Klionsky, 2009). So far, 34 ATG genes have been identified in S. cerevisiae, including ATG8, a gene with a central role in the ATG pathway (Kirisako et al., 2000; Kikuma et al., 2006; Xie et al., 2008; Noda et al., 2010). Atg8 proteins constitute one of the 62 highly conserved eukaryote-specific protein families. While yeast and other fungal species have a single ATG8 gene, multi-cellular animals, green plants and some protists have several (Shpilka et al., 2011).

In different organisms, Atg8p is a ubiquitin-like protein that undergoes cleavage of its C-terminal arginine residue by the cysteine protease Atg4p (Kirisako et al., 2000; Kabeya et al., 2004) exposing a C-terminal glycine residue. The C-terminally cleaved Atg8p is activated by Atg7p, an E1-like protein and is subsequently transferred to Atg3p, an E2-like enzyme, through a thioester bond. Later, Atg8p is conjugated to phosphatidylethanolamine (PE) by a covalent bond (Ichimura et al., 2000; Kirisako et al., 2000; Shpilka et al., 2011; Li and Vierstra, 2012), a reaction facilitated by the E3-like Atg12p-Atg5p complex; the conjugated Atg8p can also be de-conjugated by Atg4p (Kirisako et al., 2000). Ubiquitination by Atg8p is a key 
step in the autophagic pathway, and yeast null-mutant atg8 cannot conduct ATG (Zhang et al., 2007; Xie et al., 2008; Shpilka et al., 2011).

In the basidiomycete $M$. perniciosa, ATG may play a key role during its infection of Theobroma cacao since recycling of nutrients and differentiation are essential to successfully complete the fungal life cycle. This phytopathogenic fungus, the causal agent of witches' broom disease in T. cacao, has a hemibiotrophic life cycle, i.e., two distinct phases of growth that can also be observed in vitro: a biotrophic-like phase (monokaryotic cells) and a necrotrophic-like phase (dikaryotic cells) (Silva et al., 2002). To develop the spore-derived monokaryotic hypha, cells undergo dikaryotization, which allows further differentiation and production of basidiocarps that, by releasing basidiospores, complete the fungal life cycle (Silva et al., 2002; Scarpari et al., 2005).

Mycelia grown in different carbon sources (either glucose or glycerol) show different expression of $\mathrm{Mp} A T G 8$, and this alters the autophagy-dependent intracellular nutrient turnover. Glucose seems to trigger the expression of $\mathrm{Mp} A T G 8$, especially after mutagen-induced damage to cellular macromolecules. Also, MpATG8 is continuously expressed during the different stages preceding basidiocarp formation and during later steps of fungal differentiation (Santos et al., 2008).

Since MpATG8 gene expression has already been determined (Pungartnik et al., 2009), modeling of MpAtg8p is important to infer or verify the function of MpAtg8p, by comparison with the structures of known Atg8 proteins. When MpAtg8p is essential for ATG in M. perniciosa, the structure of the model could be useful in defining targets for new fungicidal molecules against witches' broom disease by de novo design methods (Andrade et al., 2009). The availability of genome sequences of the basidiomycete $M$. perniciosa and the ascomycete yeast $S$. cerevisiae allowed us to compare $A T G 8$-encoded protein ScAtg8p from $S$. cerevisiae with its putative homologue MpAtg8 protein of M. perniciosa.

\section{MATERIAL AND METHODS}

\section{Identity among basidiomycetes}

The sequence of the Atg8p obtained from the database of M. perniciosa of NCBI (ACD93204.1) was submitted to the BLASTp algorithm (Altschul et al., 1997) tool database, using non-redundant sequential parameter of search. From the sequences obtained, we selected those belonging to the phylum Basidiomycetes (Table 1).

\section{Alignment, template and construction of the three-dimensional (3-D) model}

Initially, the MpAtg8p sequence was subjected to the BLASTp program, restricted to the Protein Data Bank (PDB) (http://www.pdb.org/). Conserved regions and motifs were found by aligning the found template by TCOFFEE with the Atg8 protein sequences (Notredame et al., 2000). 3-D models were constructed using the SwissPdbViewer 3.7 software (Guex and Peitsch, 1997; Guex et al., 1999; Mcguffin and Jones, 2003) following a standard protocol: a) load template pdb file; b) align primary target sequence with template; c) submit modeling request to Swiss Model Server. PyMol was used for presentation. 


\section{Validation}

The structure derived from homology modeling was submitted to the validation process, using the programs PROCHECK 3.4 (Laskowski et al., 1993), ANOLEA (Atomic Non-Local Environment Assessment) (Melo et al., 1997; Melo and Feytmans, 1997), and QMEAN (Ramachandran et al., 1963) (http://swissmodel.expasy.org/).

\section{RESULTS AND DISCUSSION}

\section{Identity of the MpAtg8p sequence with Atg8p of other basidiomycetes}

With a sequence of 127 amino acids (aa), MpAtg8p has high similarity with Atg8 proteins of different species of basidiomycetes (Table 1), pointing to the highly conserved function of this protein. According to Nakatogawa et al. (2007), the aa alanine 76, jointly with seven other aa residues, Ile32, Lys48, Leu50, Arg65, Asp102, Phe104, and Tyr106, is highly conserved in Atg8p and, when modified, significantly reduces the efficacy of autophagy. The aa Tyr49, Leu50, Phe77, and Phe79 have been described as essential for the functionality of Atg8p since mutational changes at these positions affect the protein's activity. Tyr49 and Leu50 are positioned in a functional domain of Atg8p. After binding the lipid, Atg8p modifies its conformation and displays the Phe77 and Phe79 residues within a hydrophobic region, which allows interaction with Atg $4 p$, a step necessary for removal of Atg8p from the membrane, delivering it to the cytoplasm. Residues Tyr49 and Leu50 are essential for the maintenance of the autophagic route, where they are involved in the lipidation process (Kirisako et al., 2000; Sugawara et al., 2004; Ichimura et al., 2004; Amar et al., 2006; Nakatogawa et al., 2007). In MpAtg8p, all these residues are conserved (Figures 1 and 2), and we may, therefore, deduce that the similarity found in aa sequence of MpAtg8p with that of ScAtg8p yeast and the similarity in their 3-D protein structure lead to likewise similarity in cellular function (Geng and Klionsky, 2008).

Table 1. Identity of the MpAtg8 amino acid sequence with Atg8p of other basidiomycetes.

\begin{tabular}{llc}
\hline Basidiomycetes & GenBank No. & Identity with Atg8p of Moniliophthora perniciosa $(\%)$ \\
\hline M. perniciosa & ACD93204.1 & $100 \%$ \\
Coprinopsis cinerea & XP_001833603.1 & $98 \%$ \\
Laccaria bicolor & AAB53650.1 & $98 \%$ \\
Piriformospora indica & CCA70209.1 & $98 \%$ \\
Schizophyllum commune & XP_003035093.1 & $97 \%$ \\
Serpula lacrymans var. lacrymans & EGN96038.1 & $97 \%$ \\
Ustilago maydis & XP_761714.1 & $96 \%$ \\
Sporisorium reilianum & CBQ69980.1 & $96 \%$ \\
Melampsora larici-populina & EGG01516.1 & $94 \%$ \\
Mixia osmundae & GAA95663.1 & $92 \%$ \\
\hline
\end{tabular}

Preliminary results involving heterologous expression of a single copy of $M p A T G 8$ in a sporulation-deficient diploid yeast $\operatorname{atg} 8 / \operatorname{atg} 8$ null mutant showed that sporulation could be restored, indicating heterologous expression of a fully functional protein (Pereira $\mathrm{ACF}$, Pungartnik $\mathrm{C}$, and Brendel $\mathrm{M}$, unpublished results). 


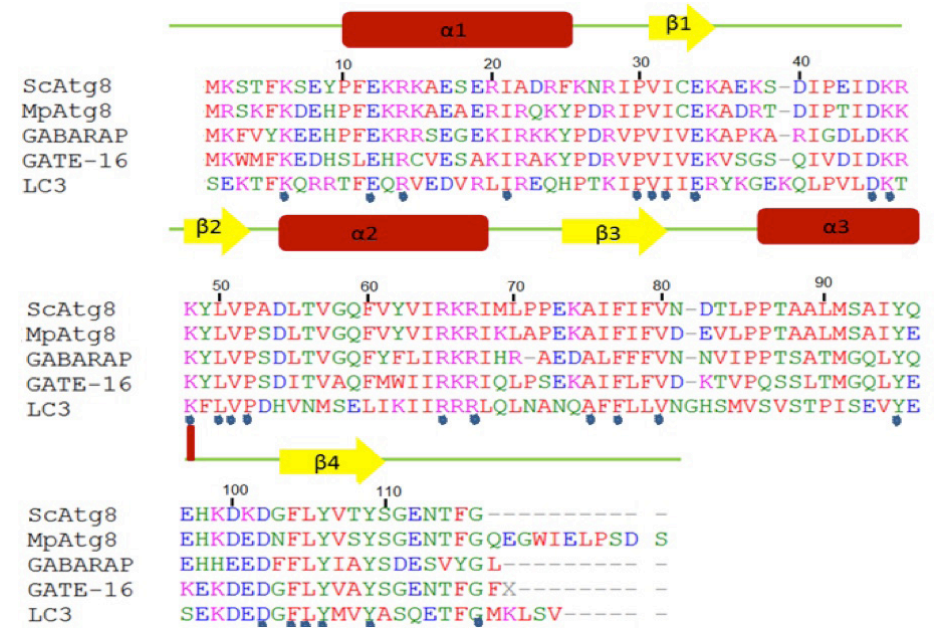

Figure 1. Multiple sequence alignment of Atg8p homologues. Amino acid sequences of Atg8p of yeast, Moniliophthora perniciosa and mammalian homologues are aligned using the Clustal_W program. Underlined residues represent conserved amino acids and asterisks mean identical amino acids in the five Atg8 proteins.

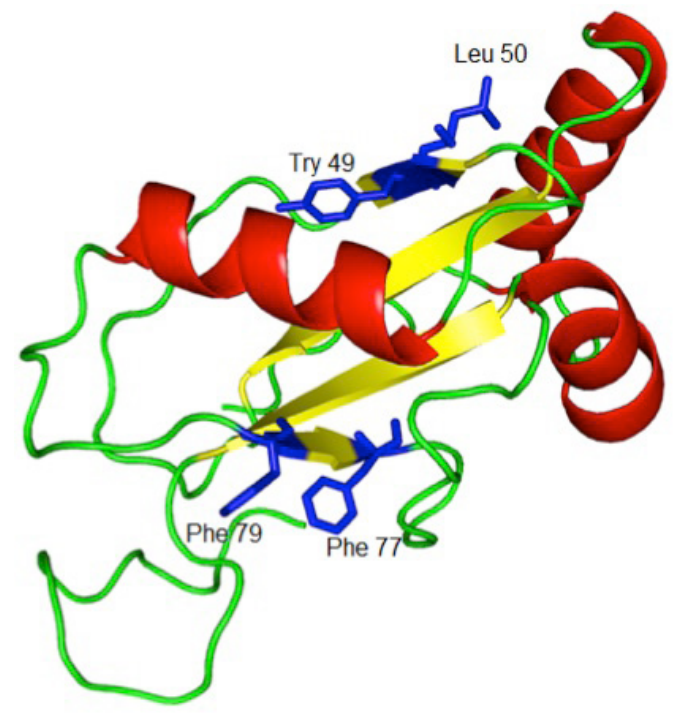

Figure 2. Three-dimensional model of MpAtg8p. Amino acids Tyr49, Leu50, Phe77, and Phe79 are marked in blue.

\section{Alignment, template and construction of the 3-D model}

The autophagic pathway of M. perniciosa is not very well described, except for some molecular data concerning protein expression (Santos et al., 2008; Pungartnik et al., 2009), in which the putative MpAtg8p is shown to be expressed during all phases of fungal growth with its induction related to oxidative stress. Therefore, the construction of a 3-D model of MpAtg8p permits the prediction of cellular function as well as provides a better understanding of domains present in the formed structure. 
Sequence similarity of aa between ScAtg8p and MpAtg8p and prediction of secondary structure is shown in Figure 1, while the 3-D structure is shown in Figure 2. All proteins classified as Atg8p have one helicoid N-terminal domain [NHD, consisting of three $\alpha$-helices, $\alpha 1$ to $\alpha 3$ ] and a second ubiquitin-like C-terminal domain [UDL, consisting of four $\beta$-sheets, $\beta 1$ to $\beta 4$ ] (Paz et al., 2000; Coyle et al., 2002; Sugawara et al., 2004; Nakatogawa et al., 2007). Similarity between the two proteins was $78 \%$ and MpAtg8p had regions characteristic of the ubiquitin family, which contain two domains consisting of three $\alpha$-helices and four $\beta$-sheets, respectively (Figure 1). After analysis by SWISS MODEL (SwissPdb Viewer v.3.7) the 3-D arrangement of both domains could be identified (Figure 2). The model of MpAtg8p features a central structure formed by four $\beta$-sheets ( $\beta 1$ to $\beta 4$ ), which is flanked by three $\alpha$-helices ( $\alpha 1$ to $\alpha 3$ ) (Figure 2). The first $\alpha$-helix in the N-terminal region of ScAtg8p (which does not belong to the ubiquitin domain) is absent in MpAtg8p (Figure 2). The main part of the structure (aa 30 to 110$)$ contains the four $\beta$-sheets ( $\beta 1-\beta 4)$ and two $\alpha$-helices $(\alpha 2$ and $\alpha 3)$ and resembles the characteristic ubiquitin structure owing to its significant aa sequence homology with the UBL protein family (Coyle et al., 2002). The $\beta 4$ sheet of MpAtg8p C-terminal region is similar to those observed in mammalian homologues of the respective Atg8p microtubule-associated protein light chain 3 [LC3], Golgi-associated ATPase enhancer of $16 \mathrm{kDa}$ [GATE-16] and receptor-associated protein [GABARAP] (Wang et al., 1999; Sugawara et al., 2004).

Alignment of the predicted MpAtg8p 3-D structure with its mammalian homologues GABARAP, LC3, and ScAtg8p yeast is shown in Figure 3. Their involvement in ubiquitination-like processes resembles that described for yeast and is similar to $\operatorname{Atg} 4 p, \operatorname{Atg} 3 p$ and Atg7p (Tanida et al., 2003; Kabeya et al., 2004; Tanida et al., 2006). When comparing the 3-D structure of our model with that of known crystals of Atg8 [ScAtg8p, LC3 and GABARAP], a similarity can be inferred (Figure 3).

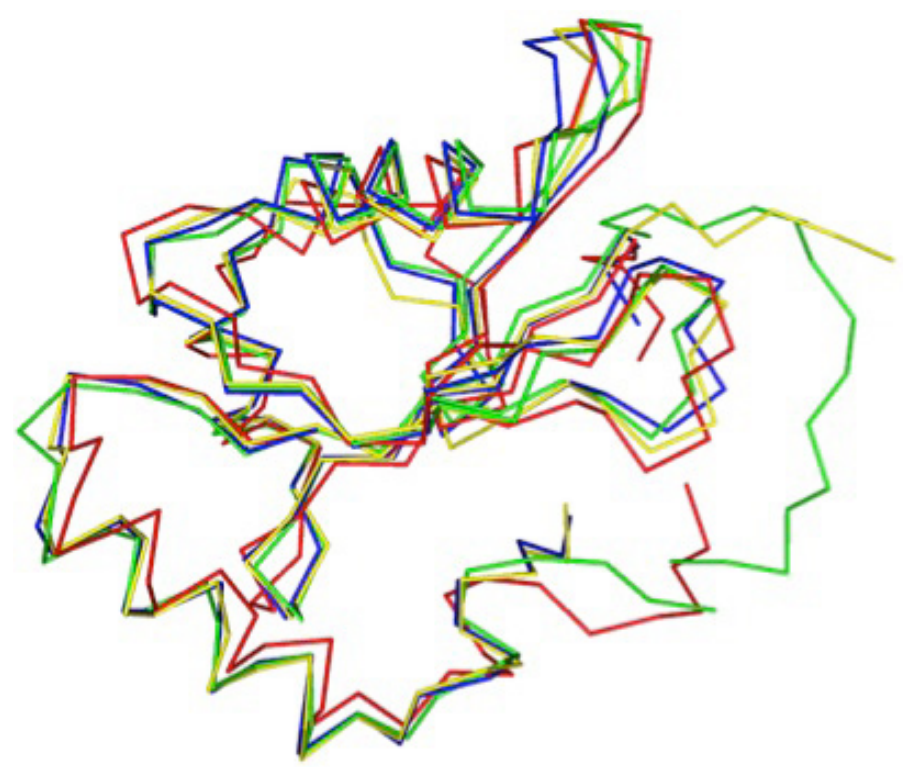

Figure 3. Alignment of the predicted MpAtg8p three-dimensional structure (red) with its mammalian homologues GABARAP (yellow), LC3 (green) and with yeast ScAtg8 (blue). 
Modeling features for the yeast ScAtg8p as well as for the proteins LC3, GATE-16 and GABARAP include one $\alpha$-helix in the first portion of the N-terminal region (Sugawara et al., 2004; Amar et al., 2006; Nakatogawa et al., 2007), whereas MpAtg8p lacks this structure (Figure 4). Compared with its homologue template (ScAtg8p), the N-terminal region of MpAtg8p contains Lys4, Asp7 and His9, differing from the ScAtg8p, which has Thr4, Ser7 and Tyr9 at the same positions. Since Nakatogawa et al. (2007) showed that deletion of the first N-terminal $\alpha$-helix of ScAtg8p does not greatly impair the functionality of autophagy in this yeast, the observed aa variations in the $\mathrm{N}$-terminus appear to be of minor influence.

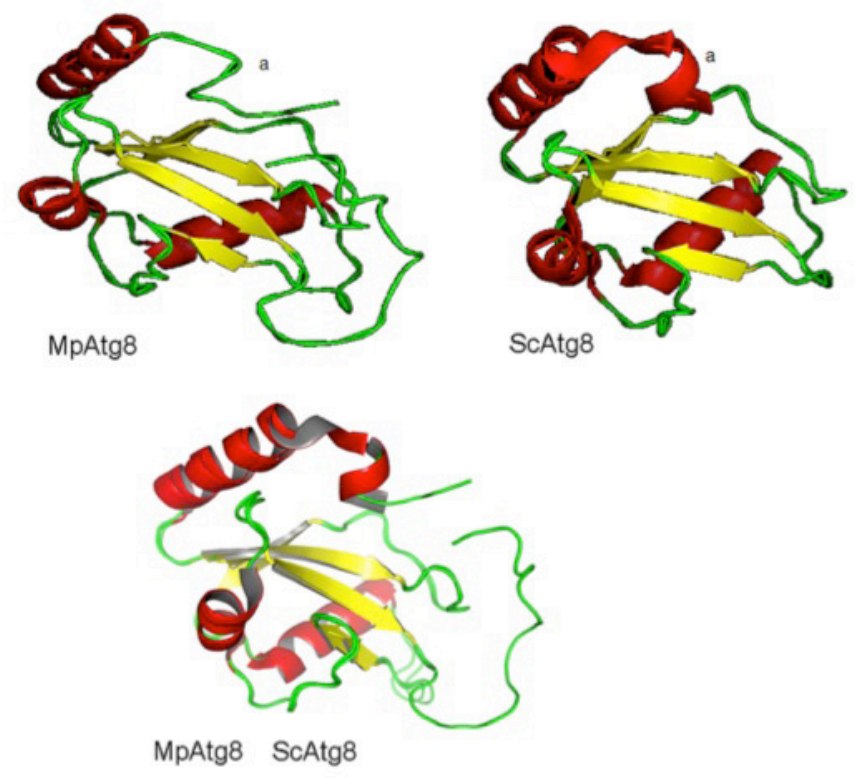

Figure 4. Structure of MpAtg8p, ScAtg8p and the three-dimensional structure of MpAtg8p/ScAtg8p alignment; "a" represents the initial N-terminal $\alpha$-helix (aa 2-9).

\section{Validation}

While the comparison of sequences allowed prediction of the probable 3-D model of MpAtg8p, in silico validation of the proposed model is required. To validate the modeling for homology, it had to be ascertained whether the main chain and the aa that compose the lateral chains are in an energy favorable position and, therefore, in suitable structural conformation, when compared to already known 3-D structures. These validation processes were carried out using the softwares QMEAN, PROCHECK 3.4 and ANOLEA (Ramachandran et al., 1963; Melo et al., 1997; Melo and Feytmans, 1997; Benkert et al., 2011; Chen et al., 2011).

The assessment with QMEAN yields values between 0 and 1, and the closer the score is to 1 the higher the model's reliability. The validation of MpAtg8p using QMEAN showed a score of 0.75 (Benkert et al., 2011), supporting the model shown in Figure 2.

The Ramachandran plot as presented by PROCHECK represents the most favored regions for an aa position (Figure 5) and the ANOLEA plot presented by PROCHECK shows 
energy distribution within MpAtg8p (Figure 6). Validation through the PROCHECK permitted the confirmation of the stereo-chemical quality of the model's structures, comparing them, aa for aa with other well-defined domain structures with a $2.0-\AA$ resolution (Pereira ACF, Pungartnik C and Brendel M, unpublished results). The product of this analysis is shown in a Ramachandran plot (Ramachandran et al., 1963) and showed that $100 \%$ of aa in our MpAtg8p model were located in energy-favorable regions (Figure 5). The distribution of aa was as follows: $93.8 \%$ in the most favored regions, $5.4 \%$ in additionally allowed favored regions, and $0.9 \%$ in generally allowed regions without any aa located in disallowed regions.

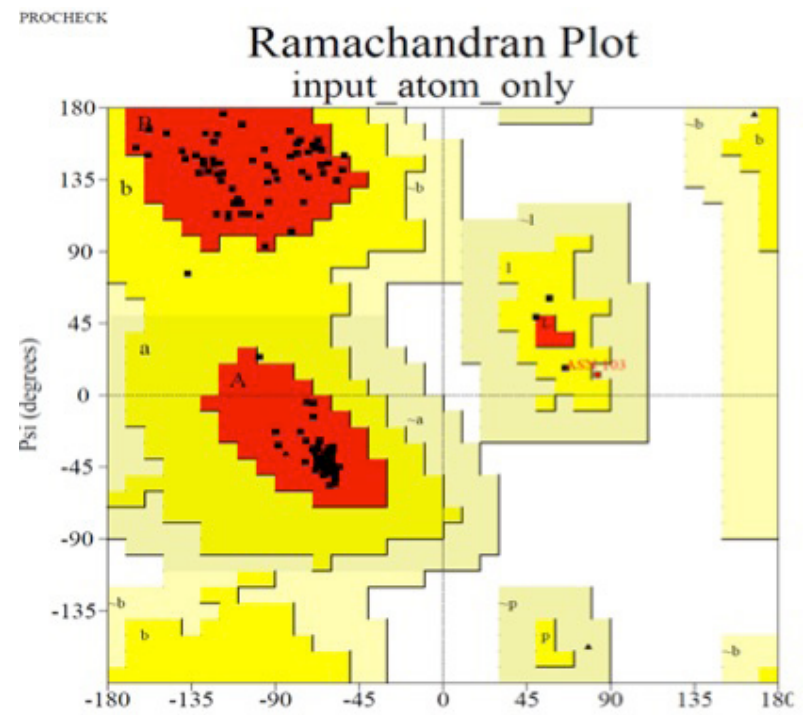

Figure 5. Ramachandran graphic as presented by PROCHECK. A, B, L (red regions) represent the most favored regions; $\mathrm{a}, \mathrm{b}, \mathrm{l}$, and $\mathrm{p}$ (yellow region) represent additionally allowed regions; $\sim \mathrm{a}, \sim \mathrm{b}, \sim 1$, and $\sim \mathrm{p}$ (light brown region) represent generally allowed regions.

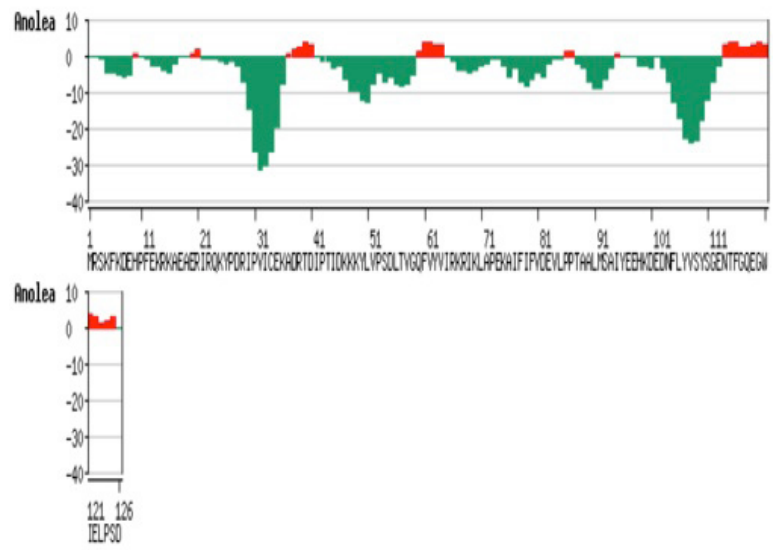

Figure 6. MpAtg8p energy. ANOLEA graphic presented by PROCHECK. The green bars represent regions of low energy. 
Evaluation of the environment of atoms weighed by means of calculations of energy allows determination of possible regions of high energy within the protein, which are related to structural instability. Structural validation by the ANOLEA program showed 105 aa with low energy and 22 with high energy and total energy of $-584 \mathrm{E} / \mathrm{kT}$, pointing to a prevalence of regions with low energy in the MpAtg8p (Figure 6). As our proposed model had a value of 0.75 , as most of the aa were located in favorable regions (Figure 5) and as our calculations did not predict high-energy but a predominance of low-energy regions (Figure 6), we are confident that it possesses structural reliability, since it was further validated by other analyses (Figures 2-4).

Prediction of the 3-D structure of MpAtg8 would provide valuable insight into the molecular basis of protein function. Here, we present the first 3-D model of the protein MpAtg8 that resembles the characteristic ubiquitin structure owing to its significant aa sequence homology with the UBL protein family. The aa sequence of MpAtg8p is highly identical with that of Atg8 proteins of other species of basidiomycetes, pointing to the conservation of this protein, and gives our model structural reliability. Since MpAtg8p may have the same function as its Atg8p homologs, this protein, essential for a functional autophagic pathway, deserves further study to perhaps serve as a new target to combat witches' broom disease.

\section{ACKNOWLEDGMENTS}

Research supported by CNPq. M. Brendel is a visiting professor at UESC. C. Pungartnik is the recipient of a CNPq fellowship. A.C.F. Pereira is the recipient of a FAPESB fellowship and data are part of her Master's dissertation at Programa de Pós-Graduação em Biologia e Biotecnologia de Micro-Organismos at UESC. We are grateful to Dr. Bruno S. Andrade for data analysis.

\section{REFERENCES}

Altschul SF, Madden TL, Schäffer AA, Zhang J, et al. (1997). Gapped BLAST and PSI-BLAST: a new generation of protein database search programs. Nucleic Acids Res. 25: 3389-3402.

Amar N, Lustig G, Ichimura Y, Ohsumi Y, et al. (2006). Two newly identified sites in the ubiquitin-like protein Atg8 are essential for autophagy. EMBO Rep. 7: 635-642.

Andrade BS, Taranto AG, Góes-Neto A and Duarte AA (2009). Comparative modeling of DNA and RNA polymerases from Moniliophthora perniciosa mitochondrial plasmid. Theor. Biol. Med. Model. 6: 22.

Benkert P, Biasini M and Schwede T (2011). Toward the estimation of the absolute quality of individual protein structure models. Bioinformatics 27: 343-350.

Chen X, Wu CW, Zhong SP, Zeng FR, et al. (2011). Molecular characterization and structure analysis of RPL10/QM-like protein from the red drum Sciaenops ocellatus (Sciaenidae). Genet. Mol. Res. 10: 576-587.

Coyle JE, Qamar S, Rajashankar KR and Nikolov DB (2002). Structure of GABARAP in two conformations: implications for GABA(A) receptor localization and tubulin binding. Neuron 33: 63-74.

Geng J and Klionsky DJ (2008). The Atg8 and Atg12 ubiquitin-like conjugation systems in macroautophagy. 'Protein modifications: beyond the usual suspects' review series. EMBO Rep. 9: 859-864.

Guex N and Peitsch MC (1997). SWISS-MODEL and the Swiss-PdbViewer: an environment for comparative protein modeling. Electrophoresis 18: 2714-2723.

Guex N, Diemand A and Peitsch MC (1999). Protein modelling for all. Trends Biochem. Sci. 24: 364-367.

He C and Klionsky DJ (2009). Regulation mechanisms and signaling pathways of autophagy. Annu. Rev. Genet. 43: 67-93.

Ichimura Y, Kirisako T, Takao T, Satomi Y, et al. (2000). A ubiquitin-like system mediates protein lipidation. Nature 408: 488-492.

Ichimura Y, Imamura Y, Emoto K, Umeda M, et al. (2004). In vivo and in vitro reconstitution of Atg8 conjugation essential for autophagy. J. Biol. Chem. 279: 40584-40592.

Kabeya Y, Mizushima N, Yamamoto A, Oshitani-Okamoto S, et al. (2004). LC3, GABARAP and GATE16 localize to autophagosomal membrane depending on form-II formation. J. Cell Sci. 117: 2805-2812. 
Kikuma T, Ohneda M, Arioka M and Kitamoto K (2006). Functional analysis of the ATG8 homologue Aoatg8 and role of autophagy in differentiation and germination in Aspergillus oryzae. Eukaryot. Cell 5: 1328-1336.

Kirisako T, Ichimura Y, Okada H, Kabeya Y, et al. (2000). The reversible modification regulates the membrane-binding state of Apg8/Aut7 essential for autophagy and the cytoplasm to vacuole targeting pathway. J. Cell Biol. 151: 263-276.

Laskowski RA, Macarthur MW, Moss DS and Thornton JM (1993). PROCHECK: a program to check the stereochemical quality of protein structures. J. Appl. Cryst. 26: 283-291.

Li F and Vierstra RD (2012). Autophagy: a multifaceted intracellular system for bulk and selective recycling. Trends Plant Sci. 17: 526-537.

McGuffin LJ and Jones DT (2003). Improvement of the GenTHREADER method for genomic fold recognition. Bioinformatics 19: 874-881.

Melo F and Feytmans E (1997). Novel knowledge-based mean force potential at atomic level. J. Mol. Biol. 267: 207-222.

Melo F, Devos D, Depiereux E and Feytmans E (1997). ANOLEA: a www server to assess protein structures. Proc. Int. Conf. Intell. Syst. Mol. Biol. 5: 187-190.

Nakatogawa H, Ichimura Y and Ohsumi Y (2007). Atg8, a ubiquitin-like protein required for autophagosome formation, mediates membrane tethering and hemifusion. Cell 130: 165-178.

Noda NN, Ohsumi Y and Inagaki F (2010). Atg8-family interacting motif crucial for selective autophagy. FEBS Lett. 584: $1379-1385$

Notredame C, Higgins DG and Heringa J (2000). T-Coffee: A novel method for fast and accurate multiple sequence alignment. J. Mol. Biol. 302: 205-217.

Paz Y, Elazar Z and Fass D (2000). Structure of GATE-16, membrane transport modulator and mammalian ortholog of autophagocytosis factor Aut7p. J. Biol. Chem. 275: 25445-25450.

Pinan-Lucarré B, Paoletti M, Dementhon K, Coulary-Salin B, et al. (2003). Autophagy is induced during cell death by incompatibility and is essential for differentiation in the filamentous fungus Podospora anserina. Mol. Microbiol. 47: 321-333.

Pollack JK, Harris SD and Marten MR (2009). Autophagy in filamentous fungi. Fungal Genet. Biol. 46: 1-8.

Pungartnik C, Melo SC, Basso TS, Macena WG, et al. (2009). Reactive oxygen species and autophagy play a role in survival and differentiation of the phytopathogen Moniliophthora perniciosa. Fungal Genet. Biol. 46: 461-472.

Ramachandran GN, Ramakrishnan C and Sasisekharan V (1963). Stereochemistry of polypeptide chain configurations. $J$. Mol. Biol. 7: 95-99.

Santos RX, Melo SC, Cascardo JC, Brendel M, et al. (2008). Carbon source-dependent variation of acquired mutagen resistance of Moniliophthora perniciosa: similarities in natural and artificial systems. Fungal Genet. Biol. 45: 851-860.

Scarpari LM, Meinhardt LW, Mazzafera P, Pomella AW, et al. (2005). Biochemical changes during the development of witches' broom: the most important disease of cocoa in Brazil caused by Crinipellis perniciosa. J. Exp. Bot. 56: 865-877.

Shpilka T, Weidberg H, Pietrokovski S and Elazar Z (2011). Atg8: an autophagy-related ubiquitin-like protein family. Genome Biol. 12: 226.

Silva SDVM, Luz EDMN, Almeida OC de, Gramacho KPG, et al. (2002). Redescrição da sintomatologia causada por Crinipellis perniciosa em cacaueiro. Agrotrópica 14: 1-28.

Sugawara K, Suzuki NN, Fujioka Y, Mizushima N, et al. (2004). The crystal structure of microtubule-associated protein light chain 3, a mammalian homologue of Saccharomyces cerevisiae Atg8. Genes Cells 9: 611-618.

Suzuki K and Ohsumi Y (2007). Molecular machinery of autophagosome formation in yeast, Saccharomyces cerevisiae. FEBS Lett. 581: 2156-2161.

Tanida I, Komatsu M, Ueno T and Kominami E (2003). GATE-16 and GABARAP are authentic modifiers mediated by Apg7 and Apg3. Biochem. Biophys. Res. Commun. 300: 637-644.

Tanida I, Sou YS, Minematsu-Ikeguchi N, Ueno T, et al. (2006). Atg8L/Apg8L is the fourth mammalian modifier of mammalian Atg8 conjugation mediated by human Atg4B, Atg7 and Atg3. FEBS J. 273: 2553-2562.

Wang H, Bedford FK, Brandon NJ, Moss SJ, et al. (1999). GABA(A)-receptor-associated protein links GABA(A) receptors and the cytoskeleton. Nature 397: 69-72.

Xie Z, Nair U and Klionsky DJ (2008). Atg8 controls phagophore expansion during autophagosome formation. Mol. Biol. Cell 19: 3290-3298.

Yorimitsu T, Zaman S, Broach JR and Klionsky DJ (2007). Protein kinase A and Sch9 cooperatively regulate induction of autophagy in Saccharomyces cerevisiae. Mol. Biol. Cell 18: 4180-4189.

Zhang Y, Qi H, Taylor R, Xu W, et al. (2007). The role of autophagy in mitochondria maintenance: characterization of mitochondrial functions in autophagy-deficient S. cerevisiae strains. Autophagy 3: 337-346. 